\title{
Density functional study of adsorption of molecular hydrogen on graphene layers
}

\author{
J. S. Arellano, ${ }^{\text {a) }}$ L. M. Molina, A. Rubio, and J. A. Alonso ${ }^{\text {b) }}$ \\ Departamento de Física Teórica, Universidad de Valladolid, 47011 Valladolid, Spain
}

(Received 10 November 1999; accepted 16 February 2000)

\begin{abstract}
Density functional theory has been used to study the adsorption of molecular $\mathrm{H}_{2}$ on a graphene layer. Different adsorption sites on top of atoms, bonds and the center of carbon hexagons have been considered and compared. We conclude that the most stable configuration of $\mathrm{H}_{2}$ is physisorbed above the center of a hexagon. Barriers for classical diffusion are, however, very small. (C) 2000 American Institute of Physics. [S0021-9606(00)70318-7]
\end{abstract}

\section{INTRODUCTION}

The adsorption of hydrogen by different forms of carbon has been studied by different groups. ${ }^{1-7}$ Dillon et al. ${ }^{1}$ were the first to study the storage of molecular hydrogen by assemblies of single wall carbon nanotubes (SWCNT) and porous activated carbon. They pointed out that the attractive potential of the walls of the pores makes it possible a high density storage. From temperature-programmed desorption experiments Dillon et al. ${ }^{1}$ concluded that those forms of carbon are promising candidates for hydrogen storage, although the density of hydrogen is still low in order to meet the requirements of the DOE Agency for novel hydrogen storage systems. More recently Levesque et al., ${ }^{2}$ Ye et al., ${ }^{3}$ and Liu et $a .^{4}$ also studied the adsorption of molecular hydrogen on SWCNT at different temperatures and pressures. Chambers et al. ${ }^{5}$ have reported obtaining an extraordinary storage capacity by some graphite nanofibers but Wang and Johnson ${ }^{6}$ have tried unsuccessfully to confirm the high storage capacity by graphite nanofibers (slit pores) and SWCNT. Hynek et al. ${ }^{7}$ investigated ten carbon sorbents but only one of them could augment the capacity of compressed hydrogen gas storage vessels. The improvement was marginal at $190 \mathrm{~K}$ and $300 \mathrm{~K}$ but nonexistent at $80 \mathrm{~K}$. The storage capacity of carbon nanotubes and graphitic fibers has been enhanced by doping with lithium and other alkali elements. ${ }^{8}$ The alkali atoms seem to have a catalytic effect in dissociating the $\mathrm{H}_{2}$ molecule and promoting atomic adsorption. An advantage is that the doped systems can operate at moderate temperatures and ambient pressure.

Some of the authors cited above $\mathrm{e}^{2,6,9}$ have also performed computer simulations of the adsorption of molecular hydrogen inside, outside and in the interstices of an array of SWCNT and in idealized carbon slit pores using model pair potentials to describe the interactions. Wang and Johnson ${ }^{6}$ adopted the semiempirical pair potential of Silvera and Goldman ${ }^{10}$ for the $\mathrm{H}_{2}-\mathrm{H}_{2}$ interaction and the $\mathrm{H}_{2}-\mathrm{C}$ interaction was modeled by a potential derived by Crowell and

\footnotetext{
a) On sabbatical leave from Area de Física, División de Ciencias Básicas e Ingeniería, Universidad Autónoma Metropolitana Azcapotzalco, Av. San Pablo 180, 02200 México D.F., México.

${ }^{b)}$ Electronic mail: jaalonso@fta.uva.es
}

Brown ${ }^{11}$ by averaging an empirical Lennard-Jones $\mathrm{H}_{2}-\mathrm{C}$ potential over a graphite plane. In the simulations Wang and Johnson used a hybrid path integral-Monte Carlo method. Johnson ${ }^{9}$ also studied the influence of electrical charging of the tubes. Stan and Cole ${ }^{12}$ performed calculations based on a sum of isotropic Lennard-Jones interactions between the molecule and the $\mathrm{C}$ atoms of the tube. They calculated the adsorption potential of a hydrogen molecule, considered as a spherically symmetric entity, as a function of distance from the axis of a SWCNT, along radial lines upon the center of a hexagon of carbon atoms and upon a carbon atom, respectively. Those simulations give useful insight to interpret the results of the experiments. However, the description of the interaction between $\mathrm{H}_{2}$ and the graphitic surfaces of the SWCNT or the slit pores in those works is too simple. Simplicity is a necessary requirement for massive simulations involving several hundred (or several thousand) $\mathrm{H}_{2}$ molecules and an assembly of SWCNT of realistic size, but one can expect more realistic results if the interaction potential is derived from an $a b$ initio calculation. The adsorption of " "atomic" hydrogen on a planar graphene sheet, that is a planar layer exfoliated from graphite, has been studied previously. ${ }^{13,14}$ Bercu and Grecu ${ }^{13}$ used a semiempirical molecular orbital LCAO treatment at the INDO (intermediate neglect of differential overlap) unrestricted Hartree-Fock level and Jeloaica and Sidis ${ }^{14}$ used the density functional formalism (DFT). ${ }^{15}$ In both works the description of the graphene layers was simplified by modeling this layer by a finite cluster $\mathrm{C}_{24}-\mathrm{H}_{12}$, where the hydrogen atoms saturate the dangling bonds on the periphery of the planar cluster. But, as mentioned above, hydrogen is adsorbed in molecular form by graphitic surfaces (SWCNT and slit pores), so in this work we study the interaction of a $\mathrm{H}_{2}$ molecule with a planar graphene layer. Since the graphene layers interact weakly in bulk graphite, the interaction of $\mathrm{H}_{2}$ with a graphitic surface is a localized phenomenon restricted to the outermost plane. For this reason our calculations have relevance for understanding the adsorption of $\mathrm{H}_{2}$ on the walls of slit pores in graphite, and also for the case of adsorption by SWCNT, since these differ from a graphene layer only in the curvature of the layer. 
TABLE I. Calculated energy (Ry) of the graphene layer for several layerlayer distances. The energies are calculated for a plane wave cut-off energy of $40 \mathrm{Ry}$.

\begin{tabular}{cc}
\hline \hline Layer-layer distance (a.u.) & Energy (per atom) \\
\hline 20 & -11.4234 \\
25 & -11.4235 \\
30 & -11.4235 \\
35 & -11.4235 \\
\hline \hline
\end{tabular}

\section{THEORETICAL METHOD AND TESTS}

To calculate the interaction between $\mathrm{H}_{2}$ and a planar graphene layer we use the $a b$ initio FHI96MD code, developed by Scheffler et al. ${ }^{16}$ This code uses the DFT ${ }^{15}$ to compute the electronic density and the total energy of the system, and we have chosen the local density approximation (LDA) for exchange and correlation. ${ }^{17}$ Only the two electrons of the $\mathrm{H}_{2}$ molecule and the four external electrons $\left(2 s^{2} 2 p^{2}\right)$ of each carbon atom are explicitly included in the calculations, while the $1 s^{2}$ core of carbon is replaced by a pseudopotential. For this purpose we use the nonlocal norm-conserving pseudopotentials of Hamann et al. ${ }^{18,19}$ Nonlocality in the pseudopotential is restricted to $l=2$, and we take as a local part of the pseudopotential the $s$ component. The code employs a supercell geometry and a basis of plane waves to expand the electronic wave functions. ${ }^{20}$

First we have tested the method for pure graphite. By minimization of the total energy with respect to the interatomic distances we obtained an in-plane $\mathrm{C}-\mathrm{C}$ bond length equal to 2.66 a.u. and a distance between planar graphitic layers of 6.275 a.u. The corresponding experimental values ${ }^{21}$ are 2.68 a.u. and 6.34 a.u., respectively. The small (1\%) underestimation of bond lengths is characteristic of the LDA. Next we have studied an isolated graphene layer. Since the computer code uses a periodic supercell method, the cell axis has to be large in the $z$-direction to avoid the interaction between graphene sheets in different cells. Table I gives the calculated energy of the graphene layer as a function of the length $c$ of the unit cell in the $z$-direction, or in other words, as a function of the distance between parallel graphene layers. Results given for $c=20,25,30$ and 35 a.u. show that the energy is well converged for those layer separations and that for $c=20$ a.u. the error in the energy per atom is only about 1 in $10^{5}$. A cutoff of 40 Ry was used in all the calculations. We also tested the method by calculating the energy of the $\mathrm{H}_{2}$ molecule, that was placed at the center of a simple cubic supercell. The total energy obtained for a plane wave cut-off energy of 40 Ry and supercell lattice constants of 18 a.u. and 20 a.u. is the same, $-2.247 \mathrm{Ry}$ as well as the bond length, 1.48 a.u. Notice that this bond length is small compared to the $\mathrm{C}-\mathrm{C}$ bond length. Anticipating the geometry to be used in the study of the interaction between $\mathrm{H}_{2}$ and graphene, another set of calculations were performed for the energy of $\mathrm{H}_{2}$ by placing the molecule in the superlattice described above in the study of the graphene layer, but this time without graphene. Calculations for distances between the imaginary graphene planes ranging from 20 a.u. to 35 a.u. (the plane wave cut-off was again $40 \mathrm{Ry}$ ) gave energies for the
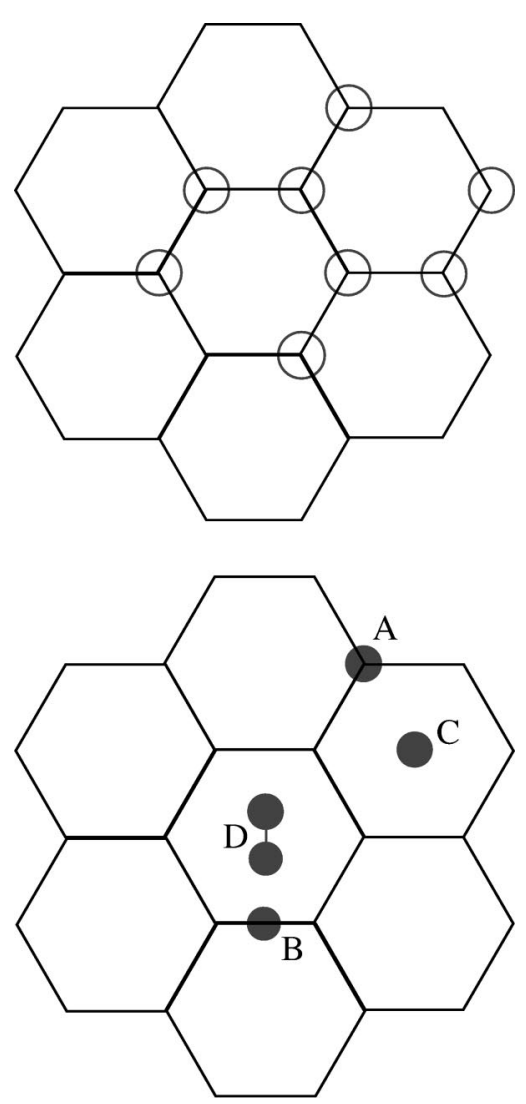

FIG. 1. The top panel gives a fragment of the graphene layer showing the eight carbon atoms in the unit cell, represented by large spheres. The bottom panel shows the three adsorption configurations with the molecular axis perpendicular to the graphene plane. These have the $\mathrm{H}_{2}$ molecule above a carbon atom (A), above the midpoint of a carbon-carbon bond (B) and above the center of a hexagon (C). Also shown is an adsorption configuration (D) with the molecular axis parallel to the graphene plane and the molecule above the center of a hexagon.

$\mathrm{H}_{2}$ molecule, identical to the energies obtained for the cubic superlattice geometry.

\section{INTERACTION BETWEEN $\mathrm{H}_{2}$ AND THE GRAPHENE LAYER}

For the periodicity of the system we have selected a unit cell with eight carbon atoms and one hydrogen molecule (see Fig. 1). If we place a hydrogen molecule at any point of the cell, the distance from this molecule to others in the nearest cells is 9.224 a.u. This separation is large compared to the bond length of $\mathrm{H}_{2}$ (1.480 a.u.), and we have verified that there is no interaction between two hydrogen molecules separated by that distance. The interaction of the $\mathrm{H}_{2}$ molecule with the graphene sheet has been studied by performing static calculations for two orientations of the axis of the molecule: axis perpendicular to the graphene plane and axis parallel to that plane. Three possible configurations, called $\mathrm{A}, \mathrm{B}$ and $\mathrm{C}$ below, have been selected for the perpendicular approach of the molecule to the plane: (A) upon one carbon atom, (B) upon the center of a carbon-carbon bond and (C) upon the center of a hexagon of carbon atoms. On the other hand, for the parallel approach the molecule is placed upon the center of a hexagon of carbon atoms with the molecular 


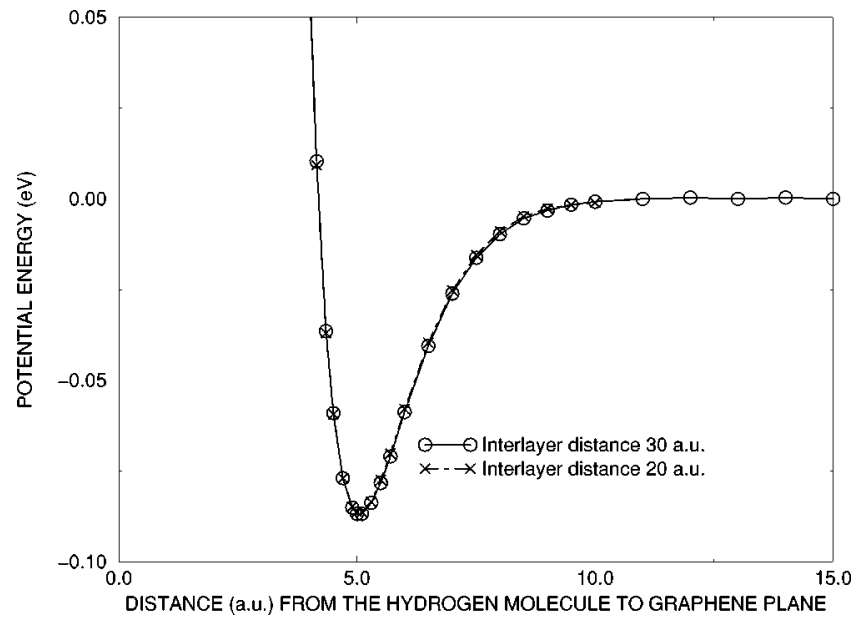

FIG. 2. A comparison of potential energy curves for the parallel approach of $\mathrm{H}_{2}$ to the graphene layer upon the center of a hexagon of carbon atoms. The curves were obtained using supercells such that the graphene layers are separated by 30 a.u. (circles) or 20 a.u. (crosses).

axis perpendicular to two parallel sides of the hexagon, and this is called configuration D. These four configurations are given in the bottom panel of Fig. 1. To obtain the interaction energy curve for each of those four cases, the distance between the hydrogen molecule and the graphene layer was varied while maintaining the relative configuration. In these calculations the bond length of the $\mathrm{H}_{2}$ was held fixed at 1.48 a.u., the bond length of the free molecule. This is expected to be valid in the relevant region of the interaction. This constraint will, however, be relaxed in simulations described at the end of this section. Calculations were first performed in the parallel configuration (D) for a superlattice such that the distance between graphene layers is 30 a.u. The plane wave cut-off was $40 \mathrm{Ry}$. The interaction energy curve is plotted in Fig. 2 and the curve has a minimum at 5.07 a.u. For separations larger than this value the energy rises fast and reaches its asymptotic value for 10-11 a.u. The energy at the maximum possible separation between the center of mass of the $\mathrm{H}_{2}$ molecule and the graphene plane for this superlattice, 15 a.u., was taken as the zero of energy. The figure also gives the results of a similar calculation for a smaller superlattice, such that the distance between graphene layers is 20 a.u. The corresponding energy curve, referred to the same zero of energy as above, is practically indistinguishable from the former curve. The calculations also show that for all practical purposes the energy curve has reached its asymptotic value for a distance of 10 a.u., that is the longest separation allowed for the superlattice of 20 a.u. This indicates that calculations using the smaller superlattice are enough for our purposes of studying the $\mathrm{H}_{2}$-graphene interaction. Then, the results of calculations corresponding to configurations $\mathrm{A}, \mathrm{B}$, $\mathrm{C}$ and $\mathrm{D}$ for a superlattice of 20 a.u. are given in Fig. 3. The potential energy curves for the perpendicular approach (A, B, C) rapidly merge with each other for large $\mathrm{H}_{2}$-graphene separation, becoming indistinguishable from one another beyond 6.5 a.u. Actually, curves A and B are very close in the whole range of separations although $\mathrm{B}$ is marginally more attractive. The common value of the energy of curves A, B and $\mathrm{C}$ at separation 10 a.u. is taken as zero of energy in Fig.

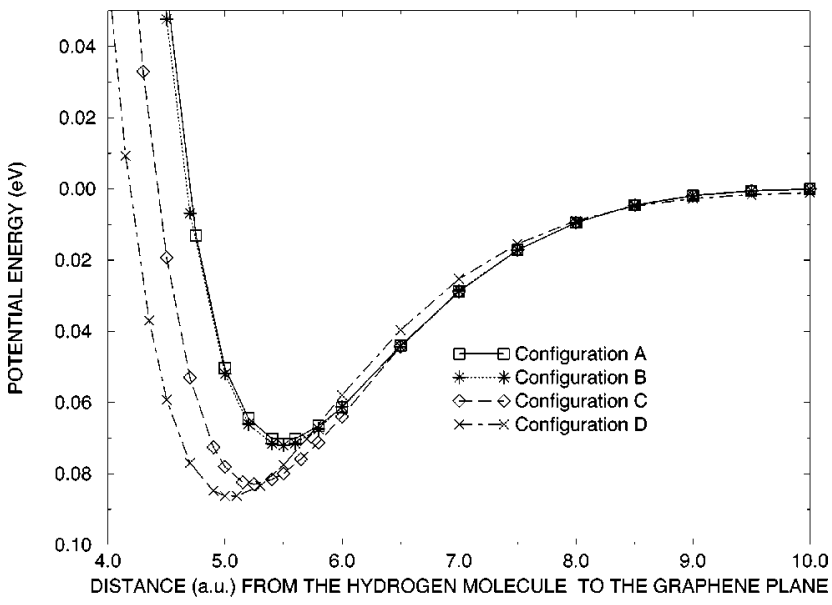

FIG. 3. Potential energy curves for the approach of $\mathrm{H}_{2}$ to the graphene layer in four different configurations. The axis of the molecule is perpendicular (A, B , C) or parallel (D) to the graphene layer. In the former orientation the molecule is above a carbon atom (A), above the center of a $\mathrm{C}-\mathrm{C}$ bond (B), and above the center of a hexagon (C). In the parallel orientation (D) the molecule is above the center of a hexagon.

3. The predicted equilibrium positions and the binding energies (depth of the minimum) of the different curves are given in Table II. The small magnitude of the binding energies, less than $0.1 \mathrm{eV}$, shows that the system is in the physisorption regime. A comparison of the four curves reveals that the most favorable position for the $\mathrm{H}_{2}$ molecule is physisorbed in a position above the center of a carbon hexagon, and that the parallel configuration is slightly more favorable than the perpendicular one. We have verified that different orientations of the molecular axis with respect to the underlying carbon hexagon in the parallel configuration lead, in all cases, to the same curve D plotted in Fig. 3. The differences in binding energy shown in Table II are very small. For instance, configurations $\mathrm{D}$ and $\mathrm{A}$ only differ by $16 \mathrm{meV}$, and configurations $\mathrm{D}$ and $\mathrm{C}$ by $3 \mathrm{meV}$.

Figures 4(a) and 4(b) give the electron density of the pure graphene layer in two parallel planes, 5 and 3 a.u. above the plane of the nuclei, respectively. The former one is very close to the preferred distance of approach for the $\mathrm{H}_{2}$ molecule in configuration D. First of all one can note that the values of the electron density in that plane are very small, of the order of $10^{-5} \mathrm{e} /(\text { a.u. })^{3}$, so the plane is in the tail region of the electron density distribution. Nevertheless the densities clearly reveal the topography of the graphene layer. Electron density contours on top of carbon atoms surround other contours representing the large hexagonal holes. Densities are

TABLE II. Binding energy (eV) and equilibrium distance (a.u.) for $\mathrm{H}_{2}$ physisorbed on a graphene layer. A, B and C correspond to the configurations in which the molecular axis is perpendicular to the graphene plane and the molecule is on top of a carbon atom (A), the midpoint of a carboncarbon bond (B) and the center of a hexagon (C). In configuration D the molecule is on top of the center of a hexagon with the molecular axis parallel to the graphene plane.

\begin{tabular}{lllll}
\hline \hline & $\mathrm{A}$ & $\mathrm{B}$ & $\mathrm{C}$ & $\mathrm{D}$ \\
\hline Binding energy & 0.070 & 0.072 & 0.083 & 0.086 \\
Distance & 5.50 & 5.49 & 5.25 & 5.07 \\
\hline \hline
\end{tabular}


(a)

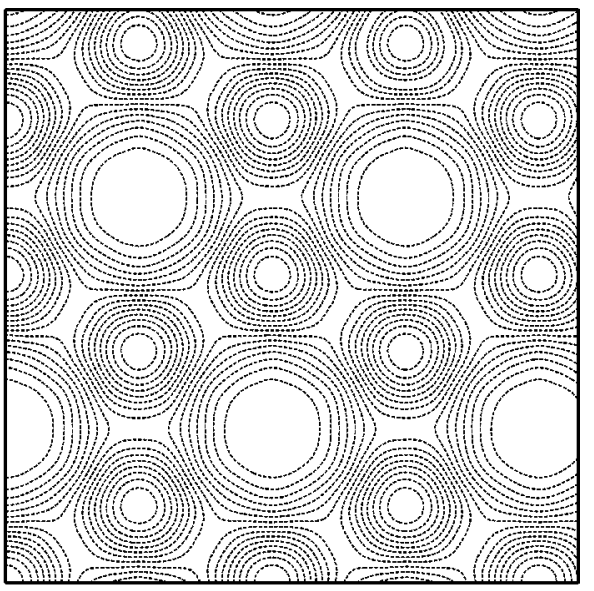

(b)

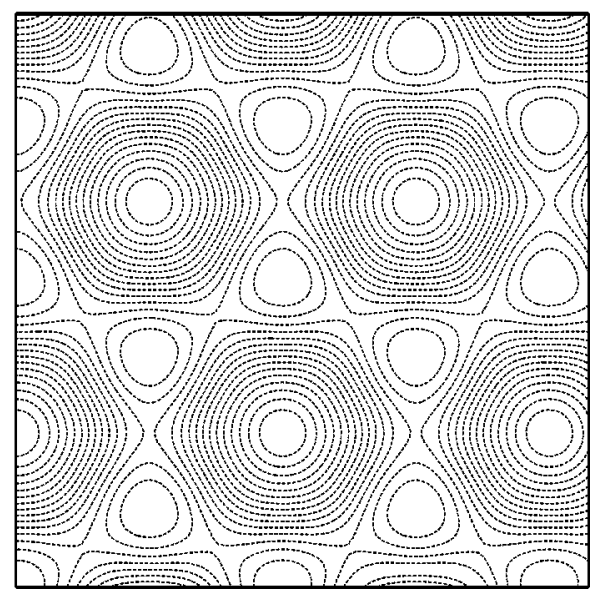

FIG. 4. (a) Contours of constant electron density $\rho$ of a pure graphene layer in a plane 5 a.u. above the plane of the carbon nuclei. $\rho=8.64$ $\times 10^{-5} \mathrm{e} /(\text { a.u. })^{3}$ in the innermost contours above carbon atoms. $\rho=6.08$ $\times 10^{-5} \mathrm{e} /(\text { a.u. })^{3}$ in the innermost contours above the large hexagonal holes. Densities decrease monotonously between those two contours with an interval $\Delta \rho=0.18 \times 10^{-5} \mathrm{e} /(\text { a.u. })^{3}$. (b) Contours in a plane 3 a.u. above the plane of carbon nuclei. $\rho=3.92 \times 10^{-3} \mathrm{e} /(\text { a.u. })^{3}$ in the innermost contours above carbon atoms. $\rho=1.72 \times 10^{-3} \mathrm{e} /(\text { a.u. })^{3}$ in the innermost contours above the large hexagonal holes. Densities decrease monotonously between those two contours with an interval $\Delta \rho=0.16 \times 10^{-3}$ e/(a.u. $)^{3}$.

larger in the other plane, closer to the plane of nuclei. In each plane the density is larger in the positions above carbon atoms and lower above the hexagons. A plot that complements this view is given in Fig. 5, that gives the electron density in a plane perpendicular to the graphene layer through a line containing two adjacent carbon atoms, labeled $C_{1}$ and $C_{2}$ in the figure. Then, points labeled $\mathrm{M}$ and $\mathrm{X}$ represent the midpoint of a carbon-carbon bond and the center of a hexagon, respectively. The most noticeable feature is the existence of depressions of electron density in the regions above the centers of carbon hexagons. These hollow regions are separated by regions of larger density that delineate the skeleton of carbon-carbon bonds. In this figure the density of the most external contour is $\rho=1.11 \times 10^{-2} \mathrm{e} /(\text { a.u. })^{3}$ and the interval between contours $\Delta \rho=1.11 \times 10^{-2} \mathrm{e} /(\text { a.u. })^{3}$.

These observations correlate with the features in Fig. 3, and lead to the following interpretation of the potential energy curves. Each curve can be seen as arising from two main contributions, one attractive and one repulsive. The at-

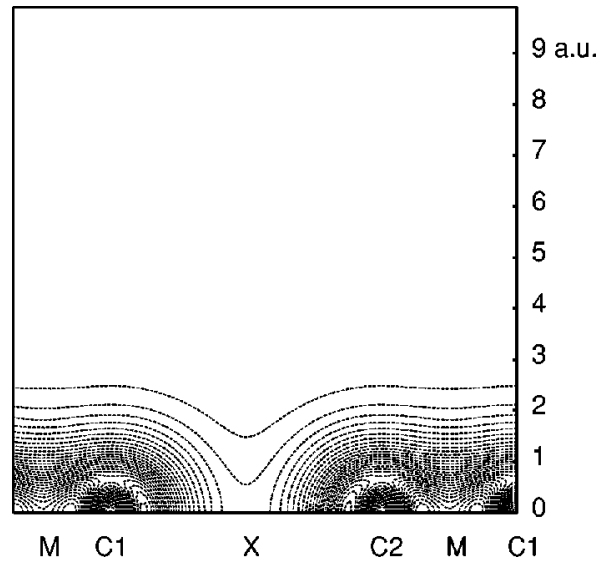

FIG. 5. Contours of constant electron density of pure graphene in a plane perpendicular to the graphene layer, going through a line containing two adjacent carbon atoms, labeled $C_{1}$ and $C_{2}$. Symbols $\mathrm{M}$ and $\mathrm{X}$ indicate the mid-point of a carbon-carbon bond and the center of a hexagon, respectively. The outermost contour plotted is $\rho=1.11 \times 10^{-2} \mathrm{e} /(\text { a.u. })^{3}$ and the interval between contours $\Delta \rho=1.11 \times 10^{-2} \mathrm{e} /(\text { a.u. })^{3}$.

tractive contribution is rather similar for all the configurations (notice the similarity of the potential energy curves beyond 6 a.u.) and is mainly due to exchange and correlation effects. Neglecting correlation for the purposes of simplicity, the exchange contribution to the total energy is given, in the LDA, by the functional

$$
E_{x}^{\mathrm{LDA}}[\rho]=C_{x} \int \rho(\mathbf{r})^{4 / 3} d^{3} r,
$$

where $C_{x}$ is a well known negative constant. ${ }^{15}$ In the regime of weakly overlapping densities, and assuming no density rearrangements due to the close-shell character of $\mathrm{H}_{2}$, the contribution of exchange to the interaction energy becomes

$$
\begin{aligned}
\Delta E_{x}= & C_{x}\left[\int\left[\rho_{\mathrm{H}_{2}}(\mathbf{r})+\rho_{g}(\mathbf{r})\right]^{4 / 3} d^{3} r\right. \\
& \left.-\int \rho_{\mathrm{H}_{2}}(\mathbf{r})^{4 / 3} d^{3} r-\int \rho_{g}(\mathbf{r})^{4 / 3} d^{3} r\right],
\end{aligned}
$$

where $\rho_{g}$ and $\rho_{\mathrm{H}_{2}}$ represent the tail densities of the graphene layer and $\mathrm{H}_{2}$ molecule, respectively. A net "bonding', contribution arises from the nonlinearity of the exchange energy functional. On the other hand the sharp repulsive wall is due to the short-range repulsion between the close electronic shell of the $\mathrm{H}_{2}$ molecule and the electron gas of the substrate. This contribution is very sensitive to the local electron density sampled by the $\mathrm{H}_{2}$ molecule in its approach to the graphene layer and explains the correlation between the position and depth of the different minima in Fig. 3 and the features of the substrate electron density in Figs. 4 and 5. Similar arguments explain the physisorption of noble gas atoms on metallic surfaces ${ }^{22}$ and the weak bonding interaction between noble gases. ${ }^{23}$ At very large separation the interaction energy curves should approach the van der Waals interaction, that is not well described, however, by the LDA.

An interesting point concerns the comparison of the minima of the curves $\mathrm{C}$ and $\mathrm{D}$ of Fig. 3. That of curve D is deeper and occurs at a shorter $\mathrm{H}_{2}$-graphene separation. The 


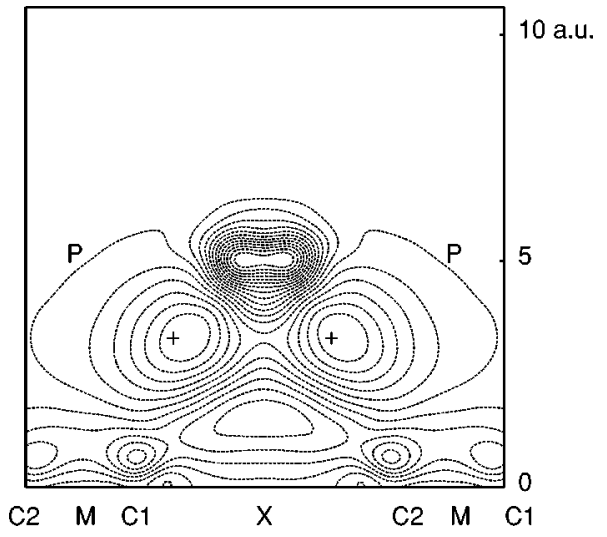

FIG. 6. Charge density difference $\rho_{\text {diff }}=\rho_{\text {tot }}-\rho_{g}-\rho_{\mathrm{H}_{2}}$ for $\mathrm{H}_{2}$ physisorbed 5 a.u. above the graphene layer. The plane of the plot and the symbols $\mathrm{C} 1, \mathrm{C} 2$, $\mathrm{M}$ and $\mathrm{X}$ are the same as in Fig. 5. The contour labeled $\mathrm{P}$ has a value $2.36 \times 10^{-5} \mathrm{e} /(\text { a.u. })^{3}$ and encloses the region of positive $\rho_{\text {diff }}$.

reason is that the surfaces of constant electron density of the $\mathrm{H}_{2}$ molecule have the shape of slightly prolate ellipsoids instead of simple spheres. Consequently, for a given distance $d$ between the center of mass of $\mathrm{H}_{2}$ and the graphene plane, the molecule with the perpendicular orientation (C) penetrates more deeply into the electronic cloud of the substrate than in the parallel orientation (D). In other words, the repulsive wall is reached earlier, that is for larger $d$, in the perpendicular configuration (C). If we consider an electronic density contour in $\mathrm{H}_{2}$ with a value $\rho=0.018 \mathrm{e} /(\text { a.u. })^{3}$, then the two semiaxes have lengths of 2.07 and 1.71 a.u., respectively, and the difference between these two lengths is 0.36 a.u. This value is in qualitative agrement with the difference between the $\mathrm{H}_{2}$-graphene separations for the two minima of curves C and D, which is 0.20 a.u. This shape effect is usually neglected in the phenomenological approaches, that treat $\mathrm{H}_{2}$ simply as a spherical molecule.

Figure 6 gives a plot of the charge density difference,

$$
\rho_{\text {diff }}(\mathbf{r})=\rho_{\text {tot }}(\mathbf{r})-\left(\rho_{g}(\mathbf{r})+\rho_{\mathrm{H}_{2}}(\mathbf{r})\right),
$$

where $\rho_{\text {tot }}(\mathbf{r})$ is the calculated density of the total system, that is the $\mathrm{H}_{2}$ molecule physisorbed in orientation $\mathrm{D}$ at a distance of 5 a.u. above the graphene layer, whereas $\rho_{g}$ $+\rho_{\mathrm{H}_{2}}$ is the simple superposition of the densities of the pure graphene layer and $\mathrm{H}_{2}$ molecule placed also in orientation $\mathrm{D}$, 5 a.u. above the graphene layer. That density difference $\rho_{\text {diff }}(\mathbf{r})$ is given in the same plane, perpendicular to the graphene layer, used in Fig. 5. $\rho_{\text {diff }}(\mathbf{r})$ has positive and negative regions. The positive region is the area bound by the contour labeled $\mathrm{P}$. This region has the shape of two lobes joined by a narrow neck. Contour P has a value $\rho_{\text {diff }}=2.36$ $\times 10^{-5} \mathrm{e} /(\text { a.u. })^{3}$ and $\rho_{\text {diff }}$ increases in this positive region as we move towards inner contours in the lobes. The innermost contour shown has a value $\rho_{\text {diff }}=2.87 \times 10^{-4} \mathrm{e} /(\text { a.u. })^{3}$. The $\mathrm{H}_{2}$ molecule sits above the neck, so the figure reveals that the repulsive interaction produced by the close electronic shell of $\mathrm{H}_{2}$ pushes some charge from the region immediately below the molecule (the neck region) to form the lobes of positive $\rho_{\text {diff }}(\mathbf{r})$. This displacement of electronic charge is nev- ertheless quantitatively very small. Notice that $\rho_{g}$ takes values between $1.6 \times 10^{-3}$ and $4.1 \times 10^{-3} \mathrm{e} /(\text { a.u. })^{3}$ in a plane 3 a.u. above the graphene layer, while $\rho_{\text {diff }}$ has values of the order $10^{-5}-10^{-4} \mathrm{e} /(\mathrm{a} . \mathrm{u} .)^{3}$ in the same plane. The smallness of $\rho_{\text {diff }}$ justifies the argument given in Eq. (2) for the attractive exchange-correlation contribution to the interaction potential.

The static calculations discussed above have been complemented with dynamical simulations in which the $\mathrm{H}_{2}$ molecule was initially placed in different orientations at distances of 4-6 a.u. from the graphene layer and was left to evolve under the influence of the forces on the $\mathrm{H}$ atoms. The $\mathrm{H}_{2}$ bond length was allowed to adjust in the process. The simulations confirm the results of the static calculations, in the sense that the $\mathrm{H}_{2}$ molecules end up in positions above the center of a hexagon at the end of the simulations. The binding energies and $\mathrm{H}_{2}$-graphene layer distances practically coincide with those in Table II. Marginally small differences in separation or binding energy are due to very small changes of the bond length of $\mathrm{H}_{2}$, always smaller than $0.3 \%$. The result of one of the simulations is worth to be mentioned. A configuration intermediate between those labeled C and D above was obtained: the center of mass of the molecule was 5.10 a.u. above the center of a carbon hexagon, with the molecular axis forming an angle of about $30^{\circ}$ with the graphene plane. The binding energy in this new configuration was only $1 \mathrm{meV}$ larger than in the parallel configuration D.

In summary, the picture arising from the calculations is rather clear. The $\mathrm{H}_{2}$ molecules prefer the hollow sites above the centers of carbon hexagons where the background electron density is lower than in channels on top of the skeleton of carbon-carbon bonds. The exchange-correlation contribution provides the weak attraction responsible for physisorption, but the preferred distance of approach is determined by the repulsive part of the interaction potential. That repulsive contribution is due to the close-shell electronic structure of $\mathrm{H}_{2}$. We have performed static calculations of the barrier for the diffusion of a molecule, initially in the parallel configuration $\mathrm{D}$ at the preferred distance of 5.07 a.u. above the graphene plane, to an equivalent configuration D above an adjacent hexagon. The initial configuration of the molecule, with its axis perpendicular to two parallel carbon-carbon bonds, can be seen in the bottom panel of Fig. 1. The molecule was then forced to follow a path across one of those bonds, allowing for the reorientation of the molecular axis at each step in order to minimize the energy of the system. Although the molecule begins with the axis parallel to the graphene plane, the orientation of the axis changes as the molecule approaches the carbon-carbon bond. In fact, when the center of mass of the molecule is precisely above that bond, the molecular axis becomes perpendicular to the graphene plane, that is the molecule adopts configuration B, as indicated also in Fig. 1. The energy difference between this saddle configuration and the starting one gives a calculated diffusional barrier of $14 \mathrm{meV}$. A temperature of $163 \mathrm{~K}$ is enough to surpass this barrier.

The conclusions from the calculations are, in our view, general enough that one can make some extrapolations to the 
case of adsorption of $\mathrm{H}_{2}$ by carbon nanotubes. When adsorption occurs on the outside wall of an isolated nanotube, the predictions of Fig. 3 will be valid, with a minor influence of the nanotube curvature. If the tubes form a parallel bundle and we consider the interstitial channels between tubes, the effects seen in Fig. 3 will be smoothed out because of the addition of contributions of different graphitic surfaces not in registry. Addition of these contributions will give rise to an interstitial channel with a potential energy nearly independent of $z$, if we call $z$ the direction parallel to the tube axis. Finally, the same smoothing effect will occur in the inner channel of a tube if the tube diameter is not large. In summary we predict very easy diffusion of the $\mathrm{H}_{2}$ molecule in arrangements of parallel tubes along the direction parallel to the tube axis, both inside the tube cavity and in the interstitial channels.

The present adsorption results can be partialy compared with those of Stan and Cole. ${ }^{12}$ They considered the $\mathrm{H}_{2}$ molecule as a spherically symmetric entity and calculated the adsorption potential inside zigzag $(13,0)$ nanotubes (radius $=9.62$ a.u.) based on a sum of isotropic Lennard-Jones interactions between the molecule and the carbon atoms of the tube. Our calculation and that of Stan and Cole agree in that the smallest binding energy is obtained for the $\mathrm{H}_{2}$ upon one carbon atom and the largest one for the $\mathrm{H}_{2}$ upon the center of the hexagon of carbon atoms. However Stan and Cole do not distinguish between parallel and perpendicular orientations because they considered a spherical molecule. Their Fig. 1 shows a binding energy about $0.079 \mathrm{eV}$ for adsorption in front of the center of a hexagon of carbon atoms and that the equilibrium distance between the molecule and the nanotube wall is 5.7 a.u. This distance is consistent but a little larger than those reported in our Table II. On the other hand, the value $0.079 \mathrm{eV}$ for the binding energy is also consistent with the binding energies in Table II. Notice, however, that the binding energy for a tube of larger radius, or for a planar graphene sheet, will be a little smaller because the curvature of the tube increases the number of nearest neighbor carbon atoms. In fact, Wang and Johnson ${ }^{6}$ calculated an adsorption binding energy near $0.050 \mathrm{eV}$ for molecular hydrogen in an idealized carbon slit pore with a pore width of 17.4 a.u.

\section{CONCLUSIONS}

By performing DFT calculations we confirm that physisorption of $\mathrm{H}_{2}$ on graphitic layers is possible. The differences between the binding energies corresponding to different positions (on top of carbon atoms, on top of carboncarbon bonds, on top of hexagonal holes) are small, and the diffusional barriers are also small, so easy diffusion is expected at low temperature. The nonsphericity of the $\mathrm{H}_{2}$ molecule has some influence on the preferred orientation of the molecular axis with respect to the graphene plane. These small effects associated to different positions and orientations of the physisorbed molecule are expected to average out inside carbon nanotubes or in the interstitial channels in parallel arrays of carbon nanotubes.

\section{ACKNOWLEDGMENTS}

Work supported by DGES (Grant No. PB95-0720-C0201), Junta de Castilla y León (Grant No. VA28/99) and European Community (TMR Contract No. ERBFMRX-CT960062-DG12-MIHT). L. M. M. is grateful to DGES for a Predoctoral Grant. J. S. A. wishes to thank the hospitality of Universidad de Valladolid during his sabbatical leave and grants given by Universidad Autónoma Metropolitana Azcapotzalco and by Instituto Politécnico Nacional (México).

${ }^{1}$ A. C. Dillon, K. M. Jones, T. A. Bekkedahl, C. H. Kiang, D. S. Bethune, and M. J. Heben, Nature (London) 386, 377 (1997).

${ }^{2}$ F. Darkrim and D. Levesque, J. Chem. Phys. 109, 4981 (1998); F. Darkrim, J. Vermesse, P. Malbrunot, and D. Levesque, ibid. 110, 4020 (1999).

${ }^{3}$ Y. Ye, C. C. Ahn, C. Witham, B. Fultz, J. Liu, A. G. Rinzler, D. Colbert, K. A. Smith, and R. E. Smalley, Appl. Phys. Lett. 74, 2307 (1999).

${ }^{4}$ C. Liu, Y. Y. Fan, M. Liu, H. T. Cong, H. M. Cheng, and M. S. Dresselhaus, Science 286, 1127 (1999)

${ }^{5}$ A. Chambers, C. Park, R. T. K. Baker, and N. M. Rodriguez, J. Phys. Chem. 102, 4253 (1998).

${ }^{6}$ Q. Wang and J. K. Johnson, J. Chem. Phys. 110, 577 (1999); J. Phys. Chem. B 103, 4809 (1999).

${ }^{7}$ S. Hynek, W. Fuller, and J. Bentley, Int. J. Hydrogen Energy 22, 601 (1997).

${ }^{8}$ P. Chen, X. Wu, J. Lin, and K. L. Tan, Science 285, 91 (1999).

${ }^{9}$ V. V. Simonyan, P. Diep, and J. K. Johnson, J. Chem. Phys. 111, 9778 (1999).

${ }^{10}$ I. F. Silvera and V. V. Goldman, J. Chem. Phys. 69, 4209 (1978).

${ }^{11}$ A. D. Crowell and J. S. Brown, Surf. Sci. 123, 296 (1982).

${ }^{12}$ G. Stan and M. W. Cole, J. Low Temp. Phys. 110, 539 (1998).

${ }^{13}$ M. I. Bercu and V. V. Grecu, Rom. J. Phys. 41, 371 (1996).

${ }^{14}$ L. Jeloaica and V. Sidis, Chem. Phys. Lett. 300, 157 (1999).

${ }^{15}$ W. Kohn and L. J. Sham, Phys. Rev. A 140, 1133 (1965); R. G. Parr and W. Yang, Density Functional Theory of Atoms and Molecules (Oxford University Press, New York, 1989).

${ }^{16}$ M. Bockstedte, A. Kley, J. Neugebauer, and M. Scheffler, Comput. Phys. Commun. 107, 187 (1997).

${ }^{17}$ J. P. Perdew and A. Zunger, Phys. Rev. B 23, 5048 (1981).

${ }^{18}$ G. B. Bachelet, D. R. Hamann, and M. Schluter, Phys. Rev. B 26, 4199 (1982).

${ }^{19}$ D. R. Hamann, Phys. Rev. B 40, 2980 (1989).

${ }^{20}$ M. C. Payne, M. P. Teter, D. C. Allan, T. A. Arias, and J. D. Joannopoulos, Rev. Mod. Phys. 64, 1045 (1992).

${ }^{21}$ M. S. Dresselhaus, G. Dresselhaus, and P. C. Eklund, Science of Fullerenes and Carbon Nanotubes (Academic, San Diego, 1996).

${ }^{22}$ N. D. Lang, Phys. Rev. Lett. 46, 842 (1981).

${ }^{23}$ R. G. Gordon and V. S. Kim, J. Chem. Phys. 56, 3122 (1972). 